Revista Brasil. Bot., V.31, n.3, p.469-489, jul.-set. 2008

\title{
Floral resources used by insects in a grassland community in Southern Brazil ${ }^{1}$
}

\author{
MARDIORE PINHEIRO ${ }^{2,5}$, BIANCA ESPINDOLA DE ABRÃO ${ }^{3}$, BIRGIT HARTER-MARQUES $^{4}$ \\ and SILVIA TERESINHA SFOGGIA MIOTTO ${ }^{3}$
}

(received: August 10, 2006; accepted: June 26, 2008)

\begin{abstract}
Floral resources used by insects in a grassland community in Southern Brazil). The goal of the present study was to identify plant species used as food source, the floral resources utilized, and the insects that visit flowers in a grassland community in southern Brazil. The study was carried out in an area of one hectare, located in a grassland formation in the Parque Estadual de Itapuã, State of Rio Grande do Sul, Brazil. The flowering pattern was seasonal, and richness and abundance of insects was higher during the period of high resource availability. Flowers of 106 species of angiosperms (73 genera and 34 families) were used as source of floral resources for 219 species ( 2,767 specimens) of insects. A total of $91.5 \%$ of plant species were visited by bees, $53.8 \%$ by flies, $34.9 \%$ by wasps, $22.6 \%$ by butterflies, and $12.3 \%$ by beetles. Nectar was the main resource consumed by the visitors (41.1\%). Asteraceae was the richest (38 spp.) and most visited family, with $63.1 \%$ of the species and $49.5 \%$ of all specimens of recorded insects. Bees were the most representative insects (33.2\% spp., $65 \%$ indiv.), followed by flies ( $26.9 \%$ spp., $16.5 \%$ indiv.), wasps, butterflies and beetles. 40 plant species were considered important resources for the floral visitors' community, due to high number of, both, species and individuals recorded in their flowers. The family Asteraceae as a species set was the main floral resource used by insect visitors through the year and has great importance for the maintenance of populations of many species of bees, flies, wasps and butterflies in the studied area.
\end{abstract}

Key words - anthophilous insects, Asteraceae, community ecology, floral resources, floral visitors

RESUMO - (Recursos florais utilizados por insetos em uma comunidade campestre no sul do Brasil). O objetivo deste trabalho foi identificar as espécies vegetais utilizadas como fontes de alimento, os recursos florais utilizados e os insetos visitantes das flores em uma comunidade campestre no sul do Brasil. O estudo foi realizado em uma parcela de um hectare, alocada em uma formação campestre no Parque Estadual de Itapuã, RS. O padrão de floração foi sazonal e a riqueza e abundância de insetos, maior no período de mais oferta de recursos. Flores de 106 espécies de angiospermas (73 gêneros e 34 famílias) foram utilizadas como fontes de recursos florais para 219 espécies e 2.767 espécimes de insetos. $91,5 \%$ das espécies vegetais foram visitadas por abelhas, $53,8 \%$ por moscas, $34,9 \%$ por vespas, $22,6 \%$ por borboletas e $12,3 \%$ por besouros. Néctar foi o principal recurso consumido pelos visitantes (41,1\%). Asteraceae foi a família mais rica (38 spp.) e a mais visitada, com $63,1 \%$ das espécies e 49,5\% de todos os espécimes de insetos registrados. Espécies com numerosas flores agrupadas em inflorescências e com recursos florais acessíveis foram as mais visitadas, por espécies e indivíduos de visitantes florais. Abelhas foram os insetos mais representativos (33,2\% spp., 65\% indiv.), seguidas de moscas (26,9\% spp., 16,5\% indiv.), vespas, borboletas e besouros. Quarenta espécies de plantas foram consideradas importantes fontes de recursos para a comunidade de visitantes florais, devido à alta abundância e elevado número de espécies de insetos registrados em suas flores. A família Asteraceae, como um conjunto de espécies, foi a principal fonte de recursos florais utilizada durante o ano, com grande importância para a manutenção das populações de muitas espécies de abelhas, moscas, vespas e borboletas ocorrentes na área de estudo.

Palavras-chave - Asteraceae, ecologia de comunidade, insetos antófilos, recursos florais, visitantes florais

\section{Introduction}

The great variety of floral types found in the Neotropical Region, demonstrates the high diversity of angiosperms

1. Part of the first author's Ph D thesis developed at the Programa de Pós-Graduação em Botânica, Universidade Federal do Rio Grande do Sul, Porto Alegre, RS, Brazil.

2. Universidade Estadual de Campinas, Departamento de Botânica, Caixa Postal 6109, 13083-970 Campinas, SP, Brazil.

3. Universidade Federal do Rio Grande do Sul, Departamento de Botânica, Av. Bento Gonçalves 9500, 91501-970 Porto Alegre, RS, Brazil.

4. Universidade do Extremo Sul Catarinense, Programa de PósGraduação em Ciências Ambientais, Av. Universitário 1105, 88806-000 Criciúma, SC, Brazil.

5._Corresponding author: mardiore.pinheiro@gmail.com in this region, and consequently a high diversity of floral visitors using resources of these plants (Endress 1994). Once the majority of angiosperms depends on pollinators for reproduction, the flower-animal interactions have a considerable influence on the biodiversity, and on the structure of plant community (Heithaus 1974, Bawa 1990), that could be characterized by the food preferences of different groups of floral visitors, the more attractive plants, and the existence of competition for resources (Barbola et al. 2000). Another important point is the necessity of understanding the plant-pollinator interactions to effective conservation and management of communities (Prance 1990).

In Brazil, there are several studies about bee-plant interactions at community level (e.g. Camargo \& 
Mazucato 1984, Martins 1995, Schlindwein 1995, Carvalho \& Bego 1997, Schlindwein 1998, Alves-dosSantos 1999, Barbola et al. 2000, Aguiar 2003, Antonini \& Martins 2003, Faria-Mucci et al. 2003, Lorenzon et al. 2003, Viana et al. 2006). However, information about floral sources for other insect groups, at community level, are less common (e.g. Silberbauer-Gottsberger \& Gottsberger 1988, Arruda \& Sazima 1996, Wilms et al. 1997, Oliveira \& Gibbs 2000, Corrêa et al. 2001, SouzaSilva et al. 2001, Darrault \& Schlindwein 2002, Machado \& Lopes 2004, Oliveira et al. 2004, Freitas \& Sazima 2006, Hermes \& Köhler 2006). Moreover, the majority of studies with anthophilous insects only indicate which plants are visited, with few works mentioning floral resources utilized in each plant species by the visitors.

The goals of the present study were to identify plant species used as food source, the floral resources utilized, and the insect visitors of the flowers in a grassland community in southern Brazil. In addition, the plant species which play a key role in the community, as resources for a high number of species and individuals of floral visitors, were also identified.

\section{Material and methods}

Study site - The present work was carried out in an area of rocky grasslands in the Parque Estadual de Itapuã (PEI) located in the South of Itapuã district $\left(30^{\circ} 20^{\prime}\right.$ and $30^{\circ} 27^{\prime} \mathrm{S}$; $50^{\circ} 50^{\prime}$ and $\left.51^{\circ} 05^{\prime} \mathrm{W}\right)$, Viamão Municipality, metropolitan region of Porto Alegre, Rio Grande do Sul State, Brazil. The total area of the PEI is about 5,566 ha (Secretaria Estadual do Meio Ambiente 1997). Data survey was performed in a plot of one hectare located in Morro do Araçá, on the west side of PEI. The vegetation in the PEI is very diverse with forests that appear mainly to the south of the mountains, rocky grasslands restricted to the top and northern slopes of hills, and with the plateau region comprising dry and wet fields, and restinga vegetation (Secretaria Estadual do Meio Ambiente 1997). These rocky grasslands are composed by scattered bushes and occasional trees, and its occurrence is related to the presence of shallow soils with low water retention capacity. According to the Köppen system (Köppen 1948), the climate in this region is classified as subtropical humid (Cfa), with well distributed rain throughout the year. Summers are usually warmer with mean temperatures in the warmest month higher than $22^{\circ} \mathrm{C}$ (Universidade Federal do Rio Grande do Sul 1982). Annual precipitation falls between 1,100 and 1,300 $\mathrm{mm}$ (Secretaria Estadual do Meio Ambiente 1997). Data recorded during the study period were obtained from the Applied Meteorology and Observation Section (Seoma) of the Eighth District of the National Institute of Meteorology (INMET), located $55 \mathrm{~km}$ from the study area.

Flowering phenologies of the species in the community were established by biweekly observations over a period of two consecutive years, between December 2002 and November 2004. Plants were grouped according flowering patterns described by Newstron et al. (1994). Floral resources utilized by visitors were verified by direct observation, and five classes of resources were defined: (1) nectar, (2) pollen, (3) nectar and/or pollen, (4) pollen and/or oil, and (5) floral tissues (petals and stamens). In classes one and two only the main used resource was considered (primary attractants, see Faegri $\&$ van der Pijl 1979). In classes 3 and 4, due to preferences for a determined resource by different groups of floral visitors, one or more resources were used in the same plant species simultaneously. In order to attribute a degree of importance as food source, each plant species was classified based on the number of species and individuals of floral visitors recorded as follow: (1) frequency of insect species: rare (1-5), frequent (5-15), very frequent (15-30); (2) frequency of insect individuals: rare (1-10), frequent (10-50), very frequent $(>50)$. Plant species were grouped into families according to Angiosperm Phylogeny Group II (2003). Voucher specimens were deposited in herbarium of Instituto de Ciências Naturais/Universidade Federal do Rio Grande do Sul.

Insects visiting the flowers were collected with entomological nets. Sampling was performed every fifteen days between December 2002 and November 2003. On each day, nine hours of sampling were divided in three periods of three hours: 8:00 to 11:00 h; 11:00 to 14:00 h, and from 14:00 to 17:00 h. During each period the plant species under observation were sampled individually, and had all floral visitors collected for 10 minutes. In each period was possible to observe four species per hour, with a total of 12 plant species in three hours. In the next two periods, the same plant species were observed again. Consequently, for each day, the same sampling effort was applied for all species observed (30 minutes each day). Thus, total sampling time for each plant species varied only with the flowering period (longer flowering periods resulted in more sampling hours), and was independent of the abundance of each species in the study area. With this method, it was possible to estimate exactly the sampling time used for each plant species through the study period. Sampling was performed by two researchers, each observing a different plant species. A total of 404 sampling hours were performed, distributed over 47 sampling days. Insect visitors were categorized into five functional groups: (1) bees, (2) wasps, (3) flies, (4) beetles, and (5) butterflies. Insect specimens were identified by specialists with identification keys and compared with specimens placed in the entomological collections from Museu de Ciências e Tecnologia/Pontifícia Universidade Católica do Rio Grande do Sul (bees), Universidade Federal de Santa Maria (wasps and flies), Museu de Ciências Naturais/Fundação Zoobotânica do Rio Grande do Sul (beetles) and Universidade Federal do Rio Grande do Sul (butterflies). Bees, social wasps (Vespidae), flies (Syrphidae) and butterflies were classified following Silveira et al. (2002), Carpenter \& Marques (2001), Marinoni et al. (2007) and Lamas (2004), respectively. Morpho-species of wasps and flies (other than social wasps and syrphids), and beetles were identified at 
family level using the keys of Triplehorn \& Johnson (2005). Collected insects were placed in the entomological collection at Museu de Ciências e Tecnologia/Pontifícia Universidade Católica do Rio Grande do Sul.

\section{Results}

In the Morro do Araçá grassland, ca. 180 plant species visited by anthopilous insects were recorded (M. Pinheiro, unpublished data), and insect visitors were collected in 106 species, representing ca. $59.0 \%$ of plant species recorded in the community. In addition, of the 64 species registered in the Asteraceae family, from rocky grasslands in Parque Estadual de Itapuã (M. E. Beretta, unpublished data), 38 species (ca. 59\%) were recorded in the present study, indicating that this sample was fairly representative of this grassland community.

Flowering pattern in the studied community was seasonal. There was a pronounced decrease in the number of flowering species during the dry season in the winter when lowest temperatures were recorded. On the other hand, an increase in the number of flowering species was observed in the beginning of the wet season, in September, increasing in October and November (figure 1-2). Among the 106 species, $67.0 \%$ presented an annual flowering pattern, $25.5 \%$ a sub annual pattern, and $7.5 \%$ a continuous flowering pattern (table 1).

During the study period, 106 species of plants belonging to 73 genera and 34 families of angiosperms were visited by 2,767 floral visitors belonging to 219 insect species representing the orders Hymenoptera, Diptera, Lepidoptera and Coleoptera. The majority of plant species

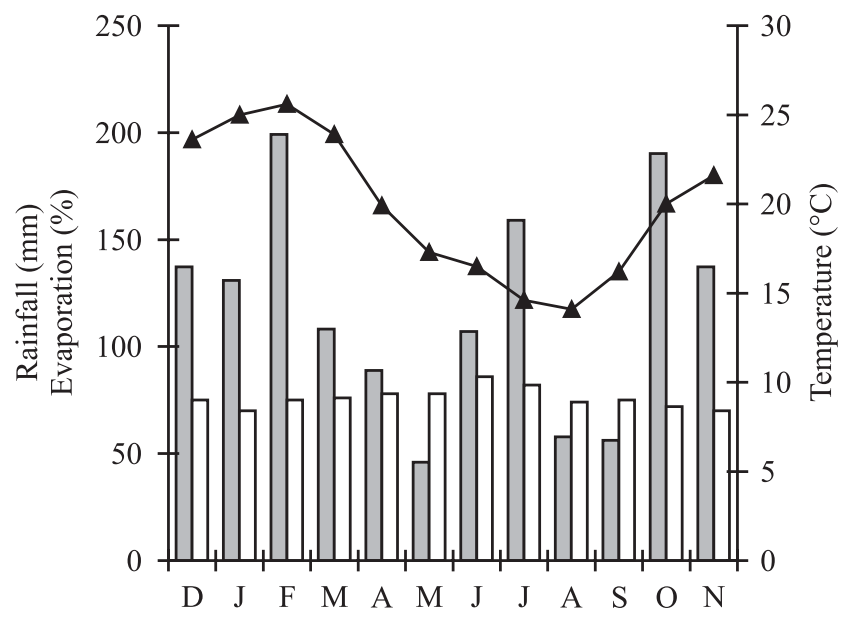

Figure 1. Diagram of climate conditions in a grassland community in southern Brazil from December 2002 to November 2003. ( $\boldsymbol{\Delta}=$ temperature; $\square=$ mean rainfall; $\square=$ evaporation).

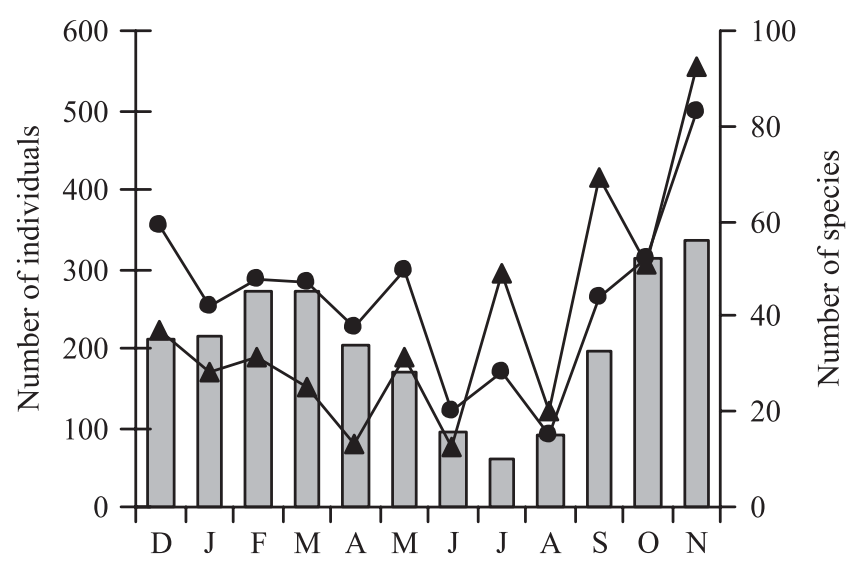

Figure 2. Number of flowering plant species and number of species and individuals of floral visitors recorded monthly from December 2002 to November 2003 in a grassland community in southern Brazil ( $\square=$ plant species; $\boldsymbol{\Delta}=$ insect species; $\bullet$ = insect individuals).

belonged to the family Asteraceae (38 spp., 35.8\%), followed by Iridaceae (8 spp.), Rubiaceae (6 spp.), Apiaceae, Myrtaceae and Verbenaceae (5 spp. each one), Oxalidaceae (4 spp.), Fabaceae and Plantaginaceae (3 spp. each one). On the other hand, 21 families were represented by only one species, and four families by two.

A total of $91.5 \%$ of plant species were visited by bees, while $53.8 \%$ were visited by flies, $34.9 \%$ by wasps, $21.7 \%$ by butterflies, and $12.3 \%$ by beetles (table 1 ). Four plant families received $75.5 \%$ of 2,767 individuals of floral visitors recorded: the family Asteraceae was the most visited with $49.5 \%$ of individuals, followed by Arecaceae (13.0\%), Apiaceae (8.0\%), and Euphorbiaceae $(5.0 \%)$ (table 1). In relation to the number of species of floral visitors, the family Asteraceae was the richest with $63.1 \%$ of 219 species of floral visitors recorded, followed by Apiaceae (23.0\%), Rubiaceae (15.2\%), Euphorbiaceae and Verbenaceae (14.3\% each one), and Arecaceae $(12.4 \%)$ (table 1$)$. The family Asteraceae also presented the highest number of species and individuals of floral visitors from each insect group, except beetles: bees (52.7\% spp., $50.3 \%$ indiv.), wasps (80.5\% spp., $59.5 \%$ indiv.), flies (62.7\% spp., $48.5 \%$ indiv.), butterflies (33.8\% spp., $59.1 \%$ indiv.), and beetle (53.8\% spp., $16.8 \%$ indv.) (table 1). At generic level Baccharis was the most visited genus, both in terms of species (37.4\%) and individuals (31.6\%) of floral visitors, followed by Butia (12.3\% spp., $13.0 \%$ indiv.), Eryngium (22.8\% spp., $8.0 \%$ indiv.), and Croton (13.6\% spp., 5.0\% indiv.) (table 1). These four genera together received $57.6 \%$ of all individuals, and $54.0 \%$ of all species of floral visitors recorded. 


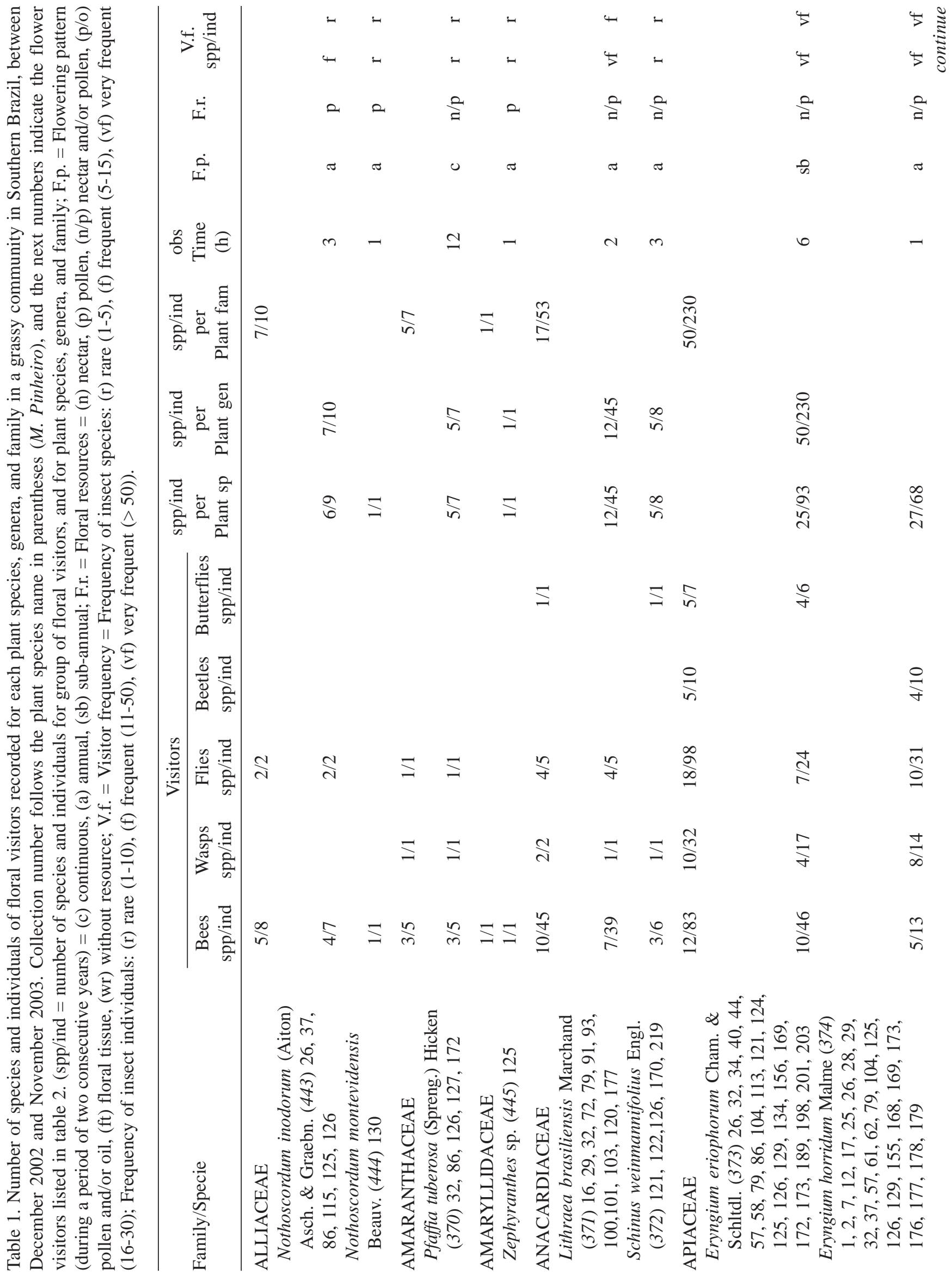




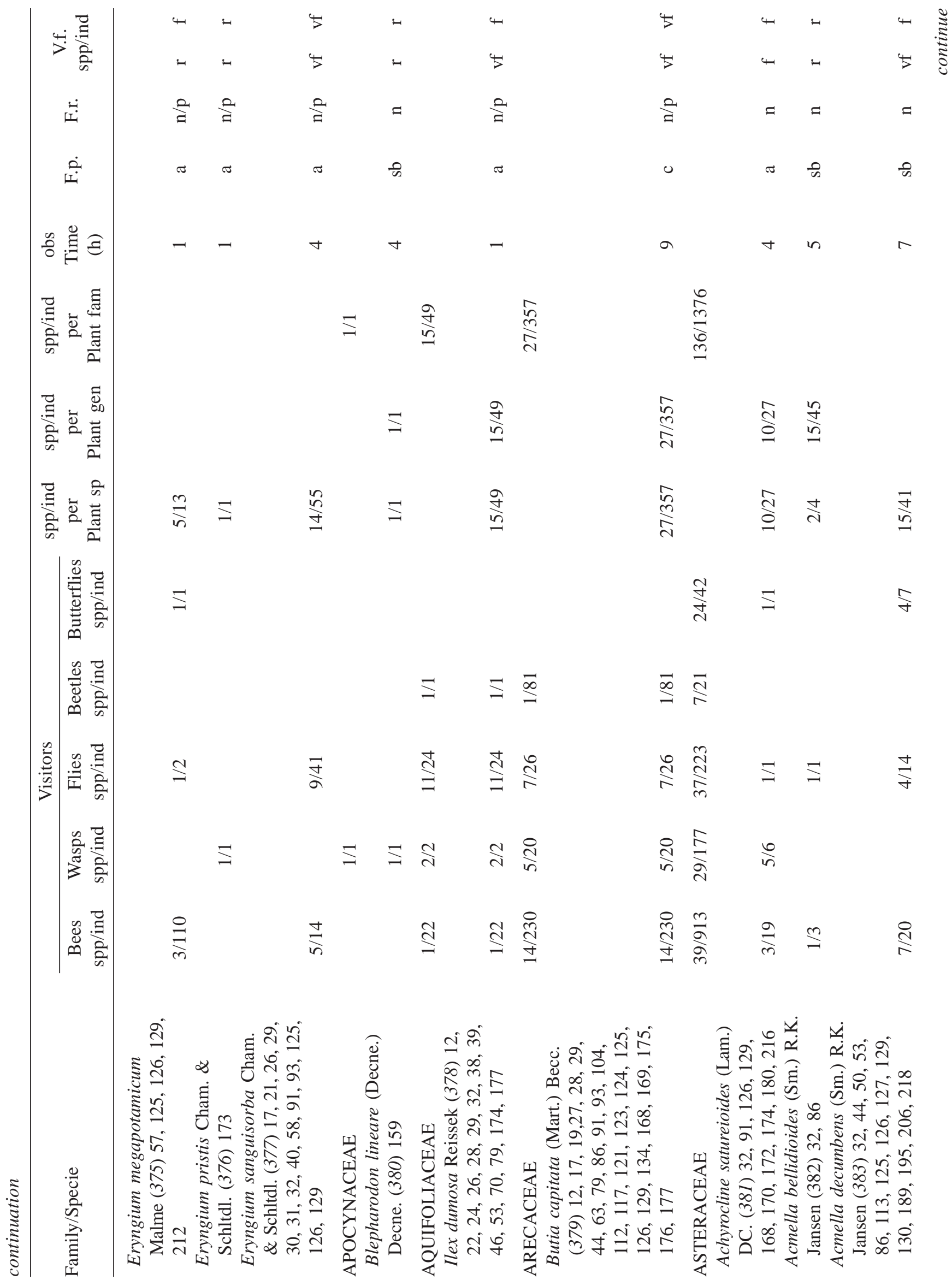


M. Pinheiro et al.: Floral resources in a grassland community

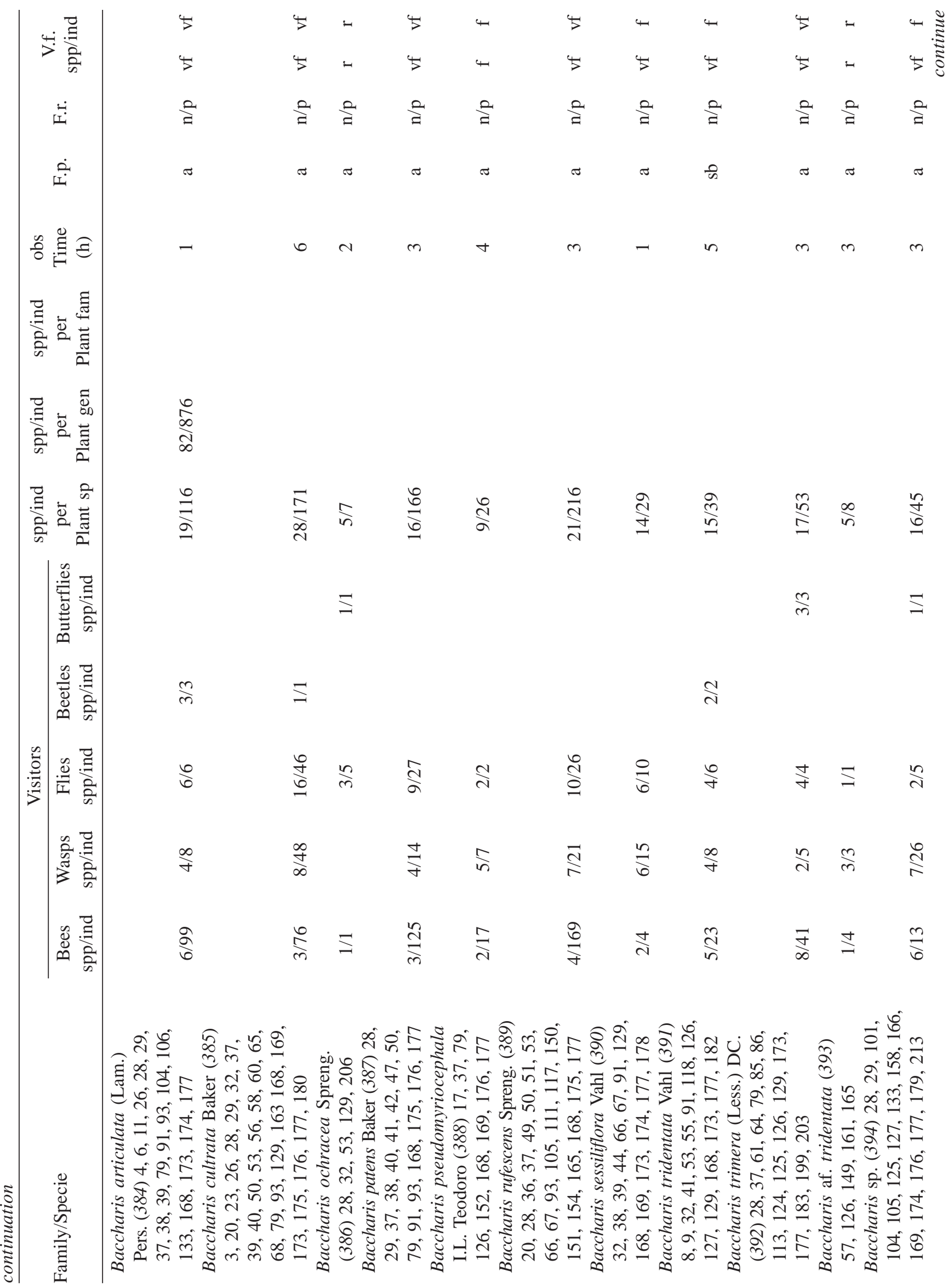




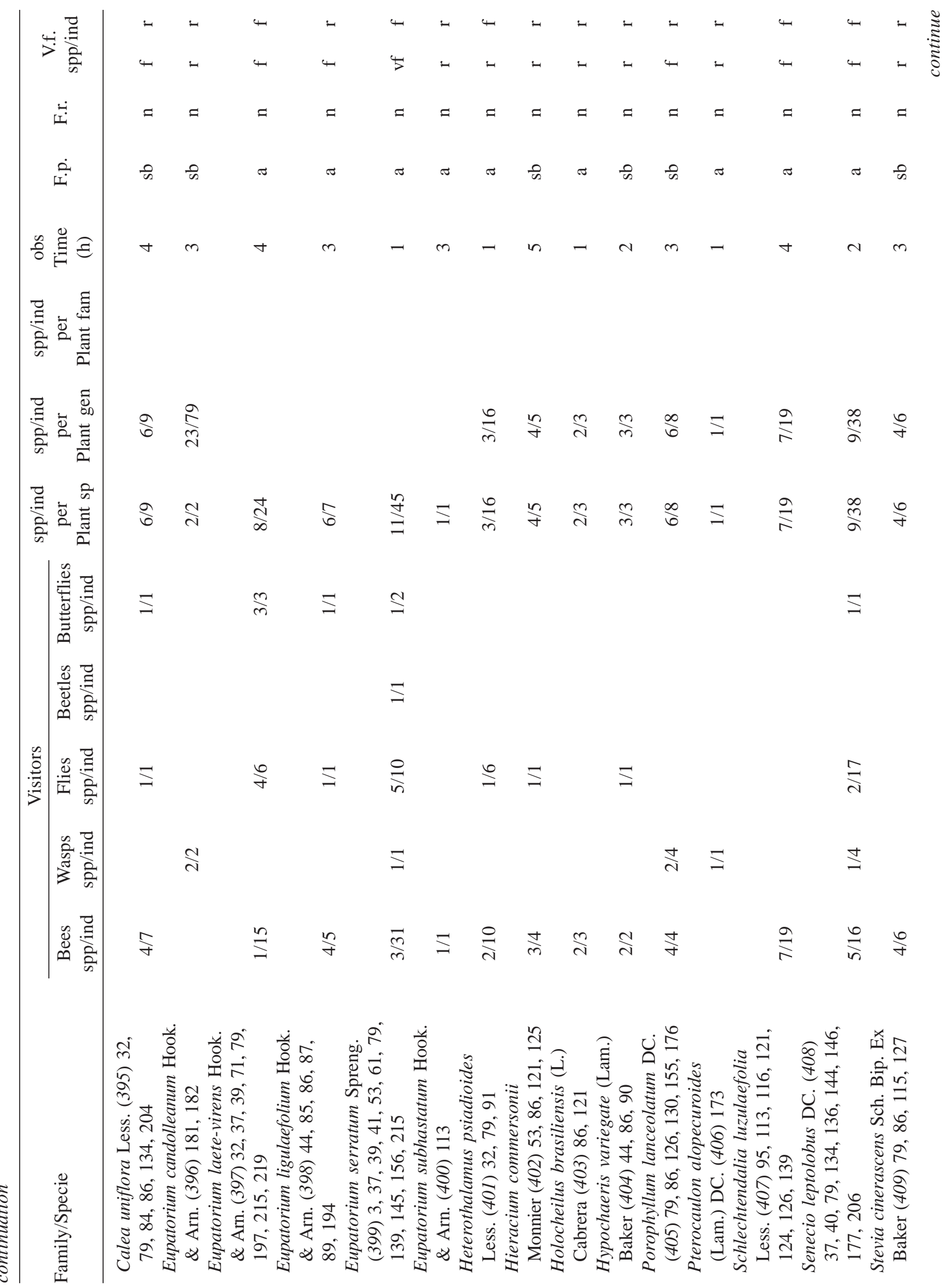




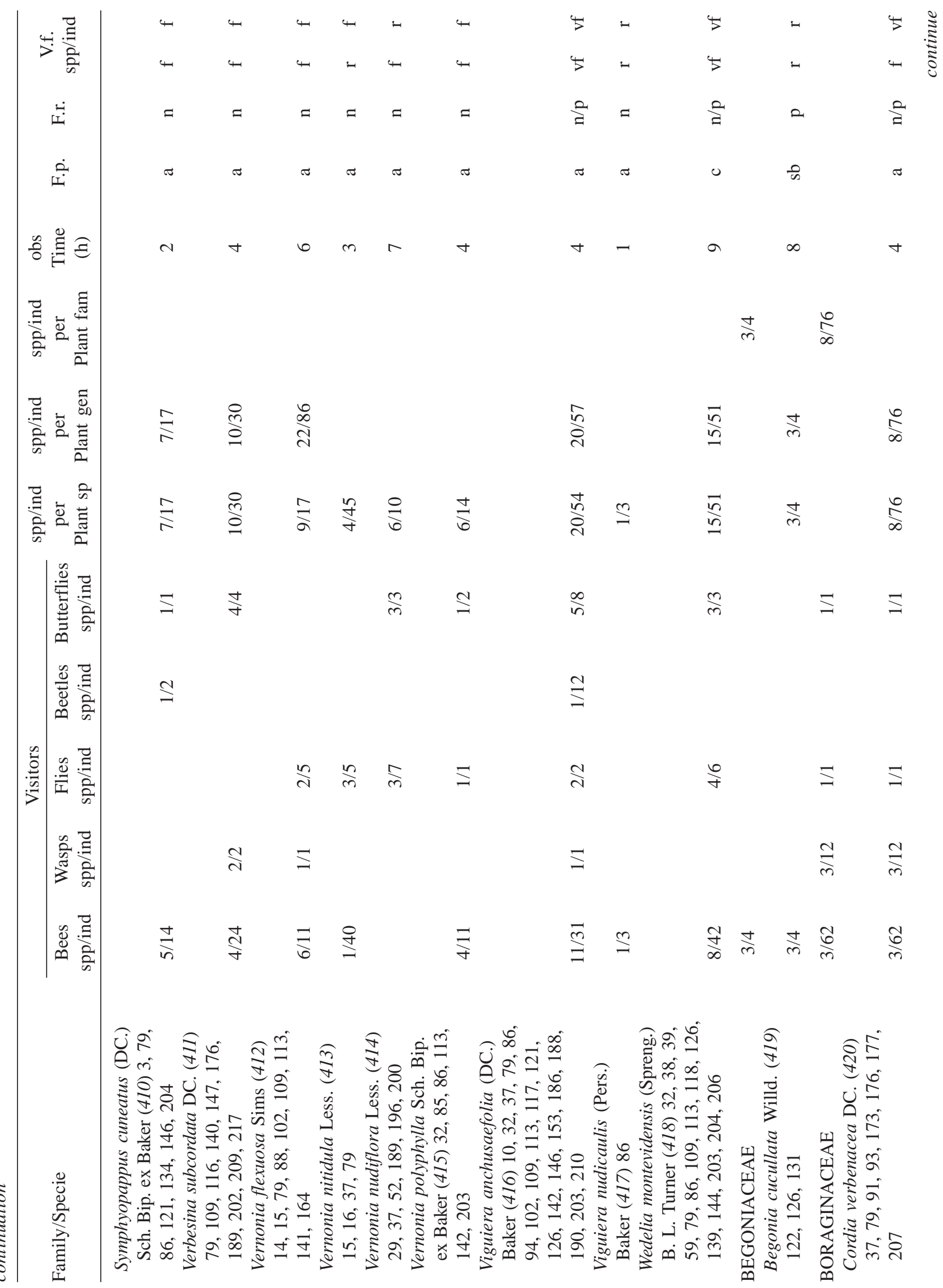


Revista Brasil. Bot., V.31, n.3, p.469-489, jul.-set. 2008

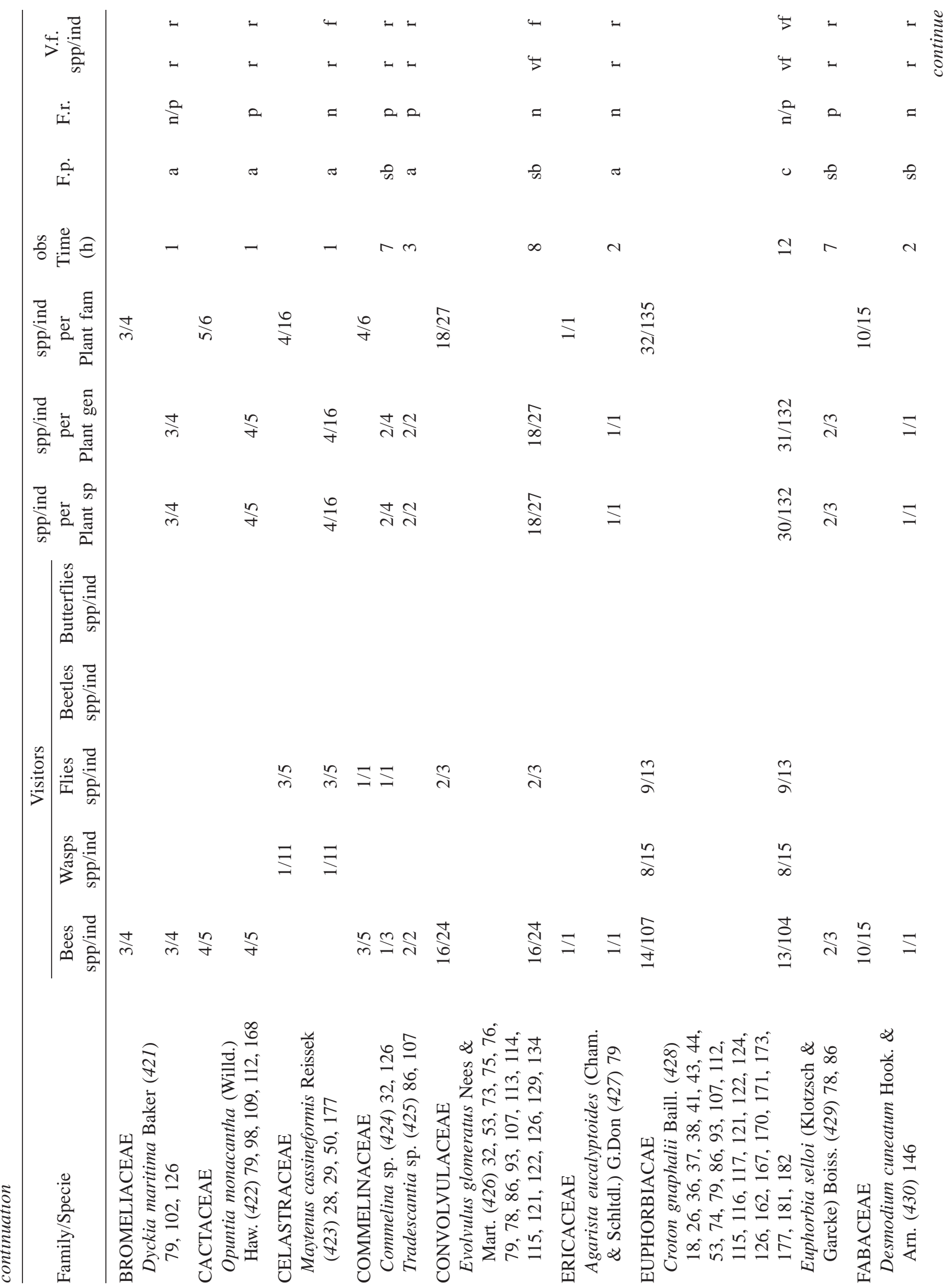




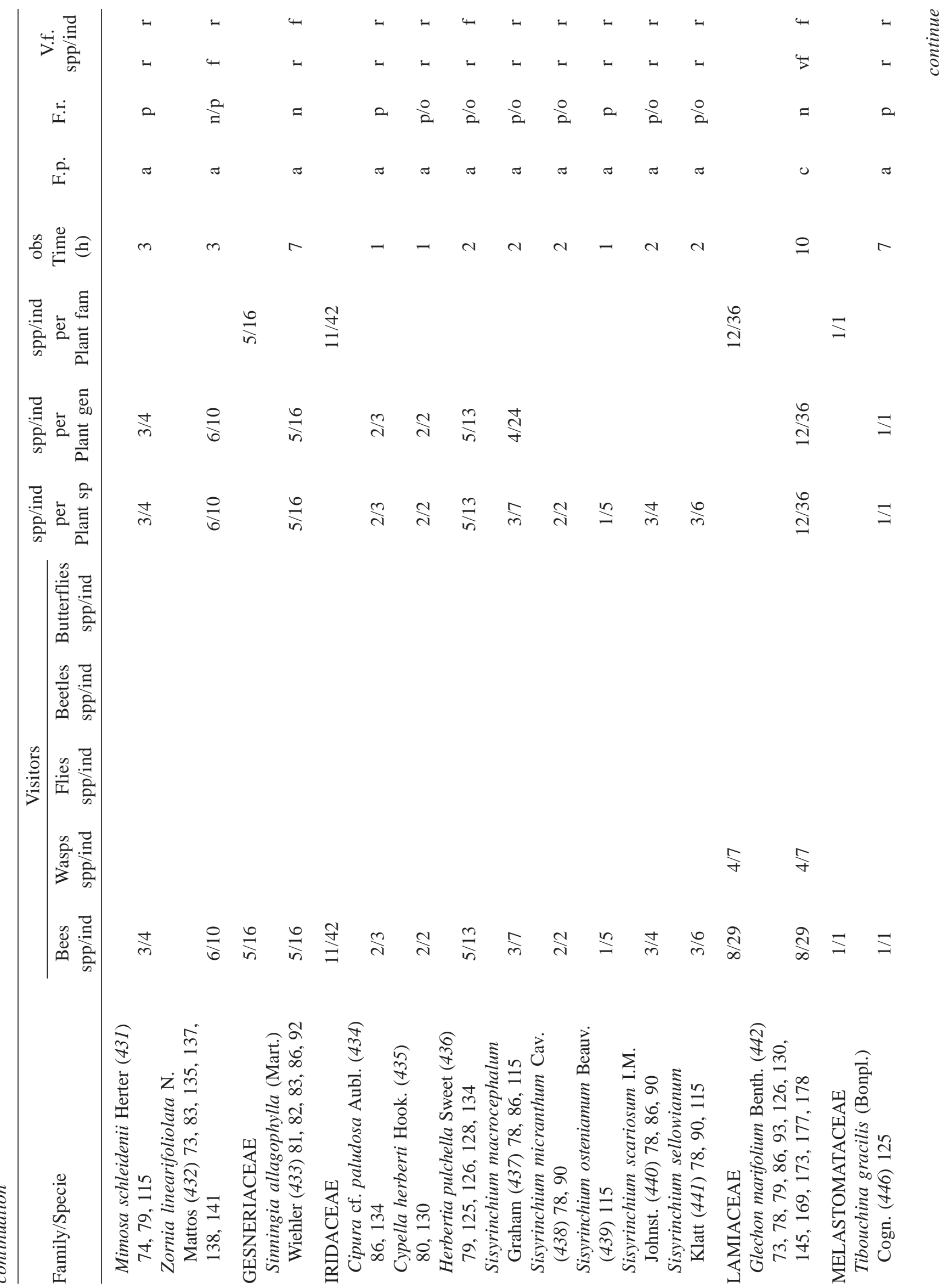


Revista Brasil. Bot., V.31, n.3, p.469-489, jul.-set. 2008

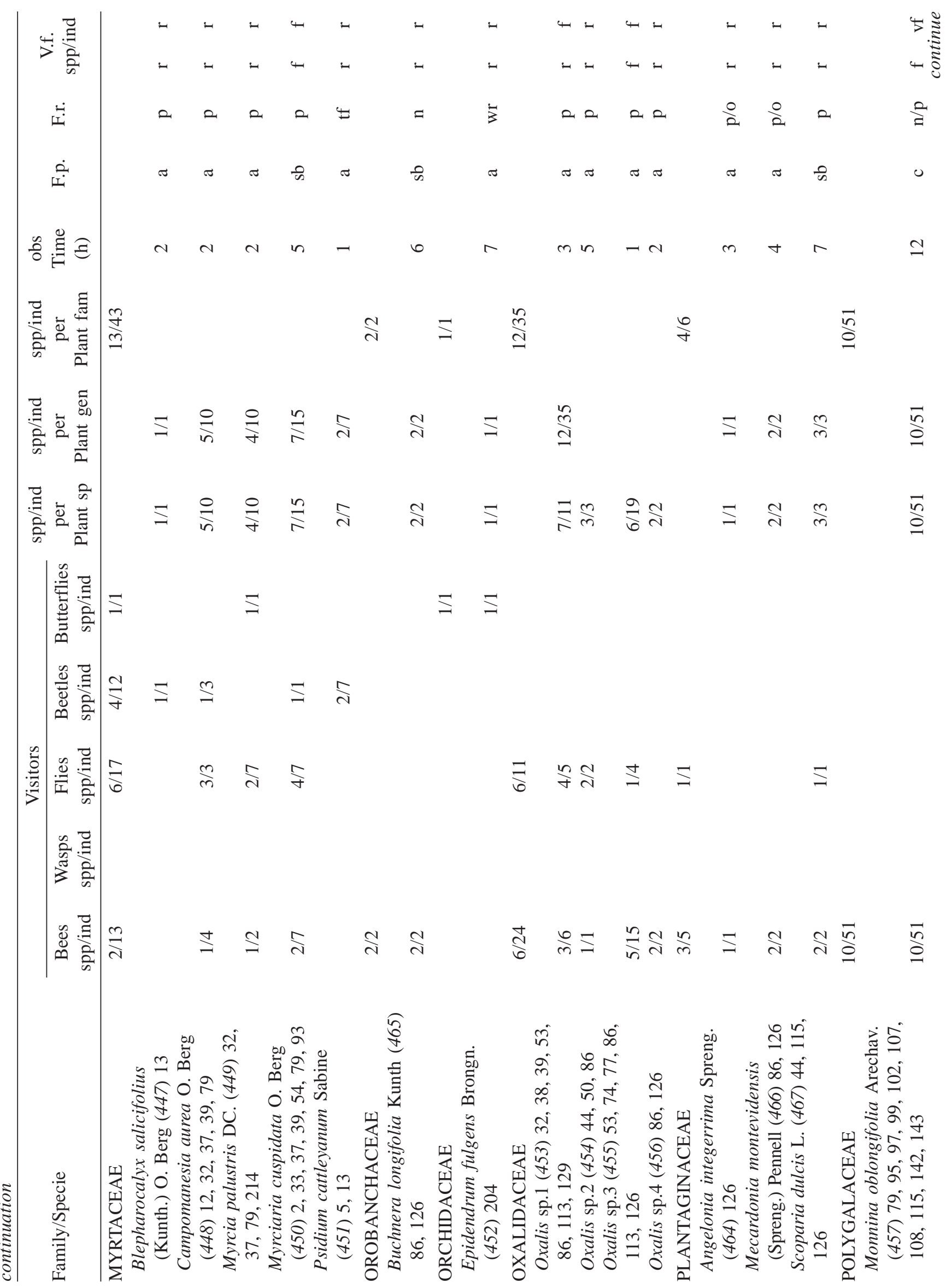


M. Pinheiro et al.: Floral resources in a grassland community

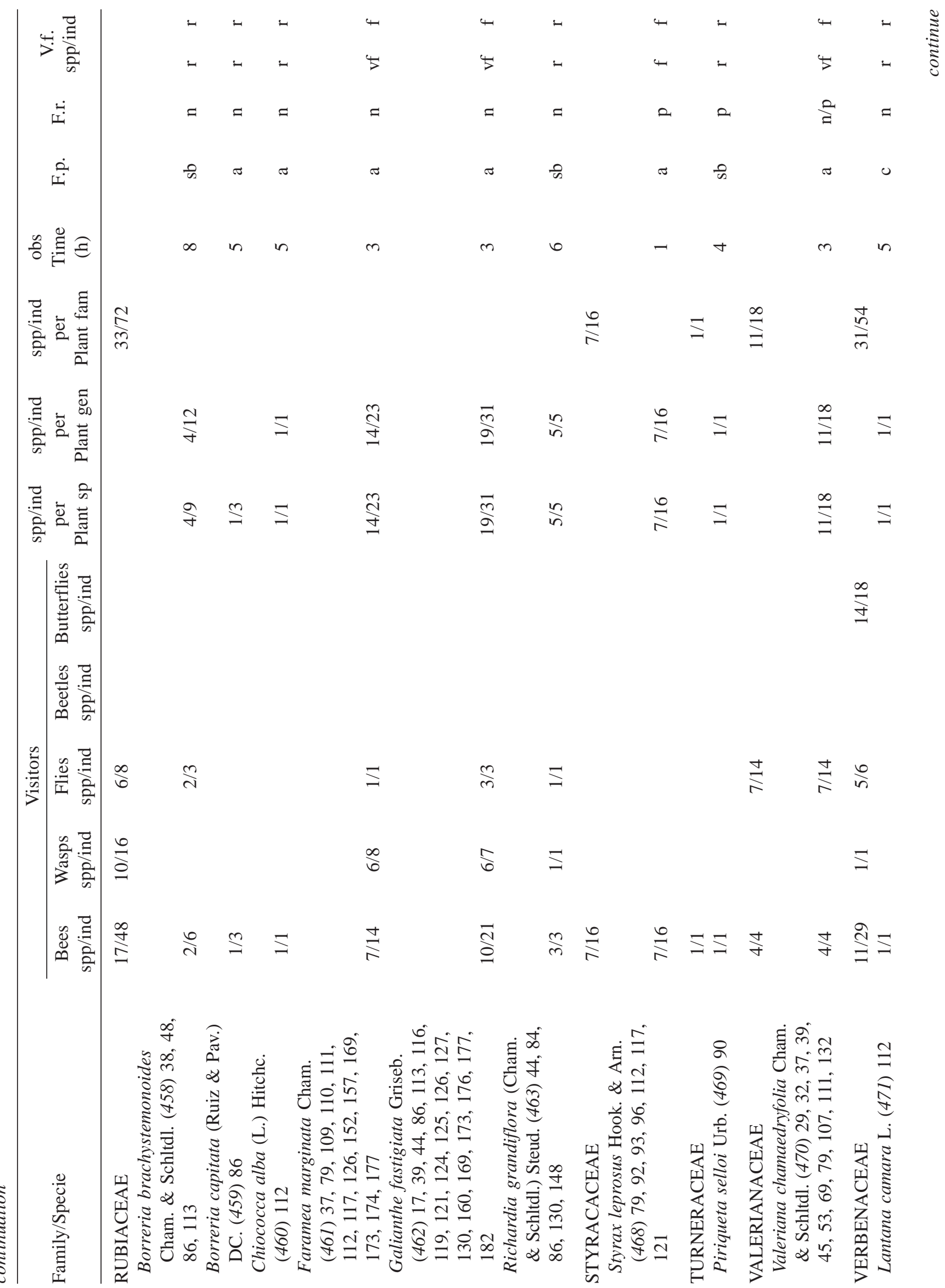




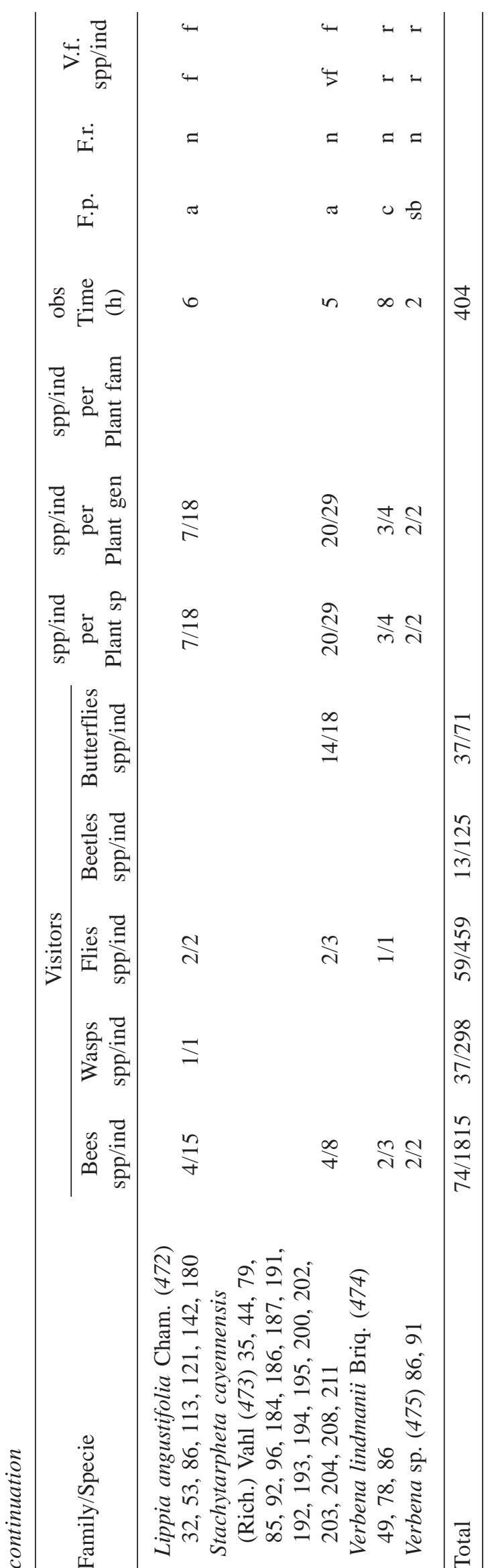

The number of visitors varied from 357 individuals recorded in flowers of Butia capitata, to a single visitor recorded in flowers of 15 plant species. The number of insect species varied from 30 species recorded in flowers of Croton gnaphalii, to one, recorded in flowers of 18 plant species. A total of $42.4 \%$ of plant species were visited by two groups of insects, while $40.6 \%$ by three groups, and $17.0 \%$ by only one. According to the frequency of floral visitors, from the total of 106 visited plant species, $56.0 \%$ and $55.7 \%$ were rarely visited by species and individuals, respectively, $19.8 \%$ and $31.1 \%$ were frequently visited by species and individuals, respectively, and $23.6 \%$ and $13.2 \%$ were very frequently visited by species and individuals, respectively. Considering the frequency of floral visitors recorded for each plant species, 40 species could be considered important resources for the floral visitors' community in the study area due to high number of, both, species and individuals recorded in their flowers (species frequently and/or very frequently visited, table 1).

Nectar was the main resource of $41.5 \%$ of plant species, followed by nectar and/or pollen $(27.3 \%)$ and pollen (21.6\%). Oil flowers were registered in eight plant species, but oil-bees were recorded in only three species. Floral tissues were consumed in only one species of Myrtaceae (table 1).

During the study period, a wide array of flower visitors was recorded on flowers. There was a pronounced decrease in the number of species of floral visitors during the dry season in the winter when lowest temperatures were recorded (figure 1-2). In this season, bee species were less abundant than wasps and flies, while beetles and butterflies were not recorded. On the other hand, an increase in the number of species of floral visitors was observed in the beginning of the wet season, in September, with a high number of species recorded during the warmest period of the year (figure 1-2). Overall, the number of individuals recorded followed the same pattern observed for the number of species, except in July when an increase in the number of individuals was recorded by the sampling of many specimens of Trigona spinipes.

A total of 1,815 bees belonging to 74 species were collected. Among the five bee families, Apidae (18 spp., $N=1,268$ ) and Halictidae (26 spp., $N=433$ ) presented the highest number of species and individuals of floral visitors, followed by Colletidae (12 spp., $N=49$ ), Megachilidae (12 spp., $N=31$ ), and Andrenidae (6 spp., $N=34$ ) (table 2). The majority of bee individuals recorded belonged to social bees Apis mellifera (23.0\%), Trigona spinipes (21.0\%), and Plebeia emerina (13.0\%). Flies were the second group of floral visitor most rich and abundant on flowers, represented by 12 families, 59 species 
and 459 individuals. Syrphidae was the predominant family (23 spp., $N=277$ ) and some species mainly in the genera Allograpta, Palpada, Pseudodorus and Toxomerus that feed on nectar and pollen were recorded in different plant species (table 2). Other abundant flies in search of nectar were represented by Muscidae, Sarcophagidae and Tachinidae. Wasps that feed on nectar were represented by eight families, 36 species and 297 individuals. The family Vespidae was the most representative (16 spp., $N=272$ ) (table 2). Some social species in this family, represented by the genera Brachygastra, Mischocyttarus, Polistes and Polybia, showed high abundance of individuals that visited several plant species (table 2). Species of beetles representing eight families (13 spp., $N=125$ ) were recorded (table 2). Almost all species of beetles were represented by few individuals, and the number of visited plant species was consequently low. In this group of floral visitors, Oedemeridae sp. 1 was responsible for $70.0 \%$ (87) of all individuals recorded, and was observed, almost exclusively, feeding on nectar and pollen on flowers of Butia capitata (81). Twelve individuals of a special genus of beetle, Nemognatha nigrotarsata (Meloidae), which has its mouth-parts adapted to form a slender tube (12 mm long) to reach deep-seated nectar (Ennes 1956, Proctor et al. 1996), were recorded. Seven families of butterflies (37 spp., $N=71$ ) were recorded (table 2). The families Hesperidae (15 spp., $N=27$ ) and Nymphalidae ( 8 spp., $N=23$ ) were predominant. Although this floral visitors represented about $17.0 \%$ (37) of all insect species recorded in the present study, few individuals of each species were collected, thus the diversity of plant species visited by each species of butterfly was low (table 2 ).

Table 2. Flower visitors recorded in a grassy community in Southern Brazil, between December 2002 and November 2003. In parentheses: (number of insect specimens/number of plant species visited).

\begin{tabular}{cl}
\hline $\begin{array}{c}\text { Species } \\
\text { number }\end{array}$ & Flower visitors \\
\hline & COLEOPTERA \\
1 & Buprestidae sp. 1 (1/1) \\
2 & Cantharidae sp. 1 (7/2) \\
3 & Cantharidae sp. $2(4 / 3)$ \\
4 & Cantharidae sp. 3 $(1 / 1)$ \\
5 & Chrysomelidae sp. 1 (4/1) \\
6 & Curculuonidae sp. 1 (1/1) \\
7 & Curculuonidae sp. 2(1/1) \\
8 & Elateridae sp. 1 (1/1)
\end{tabular}

continuation

\begin{tabular}{|c|c|}
\hline $\begin{array}{l}\text { Species } \\
\text { number }\end{array}$ & Flower visitors \\
\hline 9 & Elateridae sp. $2(1 / 1)$ \\
\hline 10 & $\begin{array}{l}\text { Nemognatha nigrotarsata (Fairmaire \& } \\
\text { Germain) (12/1) Meloidae }\end{array}$ \\
\hline 11 & Nitidulidae sp. $1(1 / 1)$ \\
\hline 12 & Oedemeridae sp. 1 (87/4) \\
\hline \multirow[t]{2}{*}{13} & Scarabaeidae sp. 1 (4/2) \\
\hline & DIPTERA \\
\hline 14 & Acroceridae sp. $1(4 / 1)$ \\
\hline 15 & Acroceridae sp. $2(2 / 2)$ \\
\hline 16 & Bombyliidae sp. 1 (2/1) \\
\hline 17 & Calliphoridae sp. $1(9 / 6)$ \\
\hline 18 & Calliphoridae sp. $2(2 / 1)$ \\
\hline 19 & Chamaemyiidae sp. $1(1 / 1)$ \\
\hline 20 & Empididae sp. $1(10 / 2)$ \\
\hline 21 & Muscidae sp. $1(1 / 1)$ \\
\hline 22 & Muscidae sp. $2(1 / 1)$ \\
\hline 23 & Muscidae sp. $3(1 / 1)$ \\
\hline 24 & Muscidae sp. $4(1 / 1)$ \\
\hline 25 & Muscidae sp. $5(1 / 1)$ \\
\hline 26 & Muscidae sp. $6(16 / 8)$ \\
\hline 27 & Sacrophagidae sp. $1(1 / 1)$ \\
\hline 28 & Sacrophagidae sp. $2(29 / 11)$ \\
\hline 29 & Sacrophagidae sp. $3(60 / 12)$ \\
\hline 30 & Sacrophagidae sp. $4(1 / 1)$ \\
\hline 31 & Sacrophagidae sp. $5(1 / 1)$ \\
\hline 32 & $\begin{array}{l}\text { Allograpta exotica (Wiedemann, 1830) (59/26) } \\
\text { Syrphidae }\end{array}$ \\
\hline 33 & $\begin{array}{l}\text { Copestylum compactum (Curran, 1925) (1/1) } \\
\text { Syrphidae }\end{array}$ \\
\hline 34 & Copestylum sp. 1 (1/1) Syrphidae \\
\hline 35 & Copestylum sp. 2 (2/1) Syrphidae \\
\hline 36 & $\begin{array}{l}\text { Palpada furcata (Wiedemann, 1819) (2/2) } \\
\text { Syrphidae }\end{array}$ \\
\hline 37 & Palpada sp. 1 (77/21) Syrphidae \\
\hline 38 & Palpada sp. 2 (17/8) Syrphidae \\
\hline 39 & Palpada sp. 3 (12/12) Syrphidae \\
\hline 40 & Palpada sp. 4 (13/5) Syrphidae \\
\hline 41 & Palpada sp. 5 (7/4) Syrphidae \\
\hline 42 & Palpada sp. 6 (2/1) Syrphidae \\
\hline 43 & Palpada sp. 7 (1/1) Syrphidae \\
\hline 44 & $\begin{array}{l}\text { Pseudodoros clavatus (Fabricius, 1794) } \\
\text { Syrphidae }(24 / 12)\end{array}$ \\
\hline 45 & Syrphidae sp. $1(1 / 1)$ \\
\hline 46 & Syrphidae sp. $2(1 / 1)$ \\
\hline 47 & Syrphidae sp. $3(1 / 1)$ \\
\hline 48 & Syrphidae sp. $4(1 / 1)$ \\
\hline 49 & $\begin{array}{l}\text { Syrphus phaeostigma Wiedemann, } 1830(2 / 2) \\
\text { Syrphidae }\end{array}$ \\
\hline 50 & Toxomerus politus (Say, 1823) (20/6) Syrphidae \\
\hline 51 & Toxomerus sp. 1 (2/1) Syrphidae \\
\hline
\end{tabular}

continue 
continuation

\begin{tabular}{ll}
\hline $\begin{array}{l}\text { Species } \\
\text { number }\end{array}$ & Flower visitors \\
\hline
\end{tabular}

52 Toxomerus sp. 2 (1/1) Syrphidae

53 Toxomerus sp. 3 (28/15) Syrphidae

54 Trichopsomyia sp. 1 (1/1) Syrphidae

55 Tabanidae sp. $1(1 / 1)$

56 Tachinidae sp. $1(3 / 1)$

57 Tachinidae sp. $2(6 / 4)$

58 Tachinidae sp. $3(10 / 2)$

59 Tachinidae sp. $4(1 / 1)$

60 Tachinidae sp. $5(1 / 1)$

61 Tachinidae sp. $6(3 / 3)$

62 Tachinidae sp. $7(1 / 1)$

63 Tachinidae sp. $8(2 / 1)$

64 Tachinidae sp. $9(1 / 1)$

65 Tachinidae sp. $10(2 / 1)$

66 Tephritidae sp. $1(3 / 2)$

67 Tephritidae sp. $2(2 / 2)$

68 Tephritidae sp. $3(1 / 1)$

69 Tipulidae sp. $1(1 / 1)$

70 Tipulidae sp. $2(1 / 1)$

71 Diptera sp. $1(1 / 1)$

72 Diptera sp. $2(1 / 1)$

\section{HYMENOPTERA}

\section{APOIDEA}

Anthrenoides sp. 14 (7/3) Andrenidae

Anthrenoides sp. 17 (8/3) Andrenidae

Anthrenoides sp. 18 (1/1) Andrenidae

Callonychium petuniae Cure \& Wittmann, 1990 (1/1) Andrenidae

Rhophitulus reticulates (Schlindwein \& Moure, 1998) (3/1) Andrenidae

Rhophitulus sp. 1 (14/8) Andrenidae

Apis mellifera Linnaeus, 1758 (481/40) Apidae

Arysoceble picta (Friese, 1899) (1/1) Apidae

Centris (Trachina) fuscata Lepeletier, 1841

(1/1) Apidae

Centris (Trachina) proxima Friese, 1899 (1/1)

Apidae

Centris (Hemisiella) tarsata Smith, 1874 (3/2)

Apidae

Ceratina (Crewella) asuncionis Strand, 1910

(2/2) Apidae

Ceratina (Crewella) paraguariensis Schrottky, 1907 (6/4) Apidae

Ceratina asunciana Strand, 1910 (135/41)

Apidae

Gaesischia (Gaesischia) fulgurans (Holmberg, 1903) (1/1) Apidae $\begin{array}{ll}\text { Gaesischia (Gaesischiopsis) sparsa Bréthes, } & 121 \\ 1910(1 / 1) \text { Apidae } & 122\end{array}$ Gaesischia sp. (1/1) Apidae

continuation

\begin{tabular}{|c|c|}
\hline $\begin{array}{l}\text { Species } \\
\text { number }\end{array}$ & Flower visitors \\
\hline 90 & $\begin{array}{l}\text { Lanthanomelissa clementis Urban, } 1995 \text { (6/5) } \\
\text { Apidae }\end{array}$ \\
\hline 91 & Plebeia emerina (Friese, 1900) (234/11) Apidae \\
\hline 92 & $\begin{array}{l}\text { Thygather (Thygather) analis (Lepeletier, 1841) } \\
\text { (6/3) Apidae }\end{array}$ \\
\hline 93 & $\begin{array}{l}\text { Trigona spinipes (Fabricius, 1793) (382/13) } \\
\text { Apidae }\end{array}$ \\
\hline 94 & Trophocleptria sp. (1/1) Apidae \\
\hline 95 & $\begin{array}{l}\text { Xylocopa (Neoxylocopa) augusti Lepeletier, } \\
1841(2 / 2) \text { Apidae }\end{array}$ \\
\hline 96 & $\begin{array}{l}\text { Xylocopa (Neoxylocopa) nigrocineta Smith, } \\
1854(4 / 2) \text { Apidae }\end{array}$ \\
\hline 97 & Caupolicana sp. (1/1) Colletidae \\
\hline 98 & $\begin{array}{l}\text { Cephalocolletes rugata Urban, } 1995 \text { (1/1) } \\
\text { Colletidae }\end{array}$ \\
\hline 99 & Cephalocolletes sp. (1/1) Colletidae \\
\hline 100 & Colletes cyaneus Holmberg, 1903 (1/1) Colletidae \\
\hline 101 & $\begin{array}{l}\text { Colletes furfuraceus Holmberg, } 1886 \text { (2/2) } \\
\text { Colletidae }\end{array}$ \\
\hline 102 & Colletes sp. 10 (20/4) Colletidae \\
\hline 103 & $\begin{array}{l}\text { Hexantheda enneomera Urban \& Graf, } 2000 \\
\text { (1/1) Colletidae }\end{array}$ \\
\hline 104 & $\begin{array}{l}\text { Hylaeus rivalis (Schrottky, 1906) (6/5) } \\
\text { Colletidae }\end{array}$ \\
\hline 105 & Hylaeus sp. 16 (4/2) Colletidae \\
\hline 106 & Hylaeus sp. 17 (2/1) Colletidae \\
\hline 107 & Sarocolletes sp. $6(7 / 5)$ Colletidae \\
\hline 108 & Sarocolletes sp. 7 (3/1) Colletidae \\
\hline 109 & $\begin{array}{l}\text { Augochlora (Augochlora) amphitrite (Schrottky, } \\
\text { 1909) (6/6) Halictidae }\end{array}$ \\
\hline 110 & Augochlora (Augochlora) daphinis Smith, 1853 \\
\hline
\end{tabular}

111 Augochlora (Augochlora) sp. 13 (3/3) Halictidae

112 Augochlora (Augochlora) tantilla Moure, 1943 (12/7) Halictidae

113 Augochlora (Oxystoglosella) semiramis Schrottky, 1910 (39/15) Halictidae

114 Augochlorella michaelis (Vachal, 1911) (1/1) Halictidae

115 Augochlorodes sp. 2 (24/10) Halictidae

116 Augochloropsis anisitsi (Schrottky, 1908) (6/4) Halictidae

117 Augochloropsis cupreola (Cockerell, 1900) (9/6) Halictidae

118 Augochloropsis euterpe (Holmberg, 1886) (2/2) Halictidae

119 Augochloropsis sp. 14 (1/1) Halictidae

120 Augochloropsis sp. 20 (1/1) Halictidae

121 Augochloropsis sp. 21 (30/13) Halictidae 
continuation

Species

number

123

124

125

126

127

128

129

130

131

132

133

134

135

136

137

138

139

140

141

142

143

144
Flower visitors

Caenohalictus sp. 6 (2/1) Halictidae

Ceratalictus sp. 2 (10/6) Halictidae

Dialictus sp. 4 (30/14) Halictidae

Dialictus sp. 9 (108/33) Halictidae

Dialictus parvus (Cresson, 1865) (6/6) Halictidae

Dialictus sp. 16 (1/1) Halictidae

Dialictus sp. 30 (99/14) Halictidae

Dialictus sp. $31(8 / 7)$ Halictidae

Dialictus sp. 32 (1/1) Halictidae

Dialictus travassosi (Moure, 1940) (1/1)

Halictidae

Neocorynura (Neocorynura) aenigma (Gribodo, 1894) (7/2) Halictidae

Paroxystoglossa brachysera Moure, 1960

(17/8) Halictidae

Ananthidium dilmae Urban, 1991 (2/1)

Megachilidae

Coelioxys (Glyptocoelioxys) pampeana

Holmberg, 1903 (1/1) Megachilidae

Epanthidium nectarinioides (Schrottky, 1902)

(1/1) Megachilidae

Epanthidum tigrinum (Schrottky, 1905) (2/1)

Megachilidae

Megachile (Acentron) sp. (4/3) Megachilidae

Megachile (Austromegachile) sp. (1/1)

Megachilidae

Megachile (Dactylomegachile) sp. (2/2)

Megachilidae

Megachile (Leptorachis) sp. 1 (7/4)

Megachilidae

Megachile (Leptorachis) sp. 2 (1/1)

Megachilidae

Megachile (Moureapis) sp. (2/2) Megachilidae

Megachile (cfr. Neochelinia) sp. (2/2)

Megachilidae

Megachile (Pseudocentron) sp. (6/4)

Megachilidae

\section{CHALCIDOIDEA}

Chalcididae sp. $1(1 / 1)$

Chalcididae sp. $2(1 / 1)$

Eurytomidae sp. $1(1 / 1)$

\section{ICHNEUMONOIDEA}

Ichneumonidae sp. $1(1 / 1)$

Ichneumonidae sp. 2 (1/1)

\section{SPHECOIDEA}

Sphecidae sp. $2(2 / 2)$

Sphecidae sp. $3(1 / 1)$

Sphecidae sp. $5(1 / 1)$

Sphecidae sp. $6(2 / 2)$

Sphecidae sp. $7(2 / 2)$ continuation

\begin{tabular}{|c|c|}
\hline $\begin{array}{l}\text { Species } \\
\text { number }\end{array}$ & Flower visitors \\
\hline & VESPOIDEA \\
\hline 157 & Pompilidae sp. 1 (1/1) \\
\hline 158 & Pompilidae sp. $3(2 / 1)$ \\
\hline 159 & Pompilidae sp. $4(1 / 1)$ \\
\hline 160 & Pompilidae sp. $5(1 / 1)$ \\
\hline 161 & Pompilidae sp. $6(1 / 1)$ \\
\hline 162 & Pompilidae sp. 7 (1/1) \\
\hline 163 & Pompilidae sp. $8(1 / 1)$ \\
\hline 164 & Scolliidae sp. $(1 / 1)$ \\
\hline 165 & Tiphiidae sp. $1(2 / 2)$ \\
\hline 166 & Tiphiidae sp. $2(2 / 1)$ \\
\hline 167 & Alphamenes sp. 1 (1/1) Vespidae \\
\hline 168 & $\begin{array}{l}\text { Brachygastra lecheguana (Latreille, 1824) } \\
\text { (36/11) Vespidae }\end{array}$ \\
\hline 169 & $\begin{array}{l}\text { Mischocyttarus drewseni Saussure, } 1857 \text { (19/10) } \\
\text { Vespidae }\end{array}$ \\
\hline 170 & Omicron sp. (4/3) Vespidae \\
\hline 171 & $\begin{array}{l}\text { Pachodynerus guadulpensis (Saussure, 1853) } \\
\text { (2/1) Vespidae }\end{array}$ \\
\hline 172 & Parancistrocerus sp. (3/3) Vespidae \\
\hline 173 & $\begin{array}{l}\text { Polistes billardieri ruficornis Saussure, } 1853 \\
\text { (44/14) Vespidae }\end{array}$ \\
\hline 174 & $\begin{array}{l}\text { Polistes cinerascens Saussure, } 1854 \text { (12/6) } \\
\text { Vespidae }\end{array}$ \\
\hline 175 & Polistes lanio (Fabricius, 1775) (6/4) Vespidae \\
\hline 176 & $\begin{array}{l}\text { Polybia ignobilis (Haliday, 1836) (17/10) } \\
\text { Vespidae }\end{array}$ \\
\hline 177 & $\begin{array}{l}\text { Polybia scutellaris (White, 1841) (109/20) } \\
\text { Vespidae }\end{array}$ \\
\hline 178 & Polybia sericea Oliver, 1922 (3/3) Vespidae \\
\hline 179 & Polybia sp. $1(2 / 2)$ Vespidae \\
\hline 180 & Stenodynerus sp. (4/3) Vespidae \\
\hline 181 & $\begin{array}{l}\text { Zeta argillaceum (Linnaeus, 1758) (2/2) } \\
\text { Vespidae }\end{array}$ \\
\hline 182 & $\begin{array}{l}\text { Zethus schrottkyanus (Von Ihering, 1911) (8/4) } \\
\text { Vespidae }\end{array}$ \\
\hline
\end{tabular}

\section{LEPIDOPTERA}

183 Achlyodes mithridates thraso (Hübner, [1807]) (1/1) Hesperiidae

184 Codatractus aminias (Hewitson, 1867) (1/1) Hesperiidae

185 Gorgythion begga begga (Prittwitz, 1868) (1/1)

Hesperiidae

186 Hylephila phyleus phyleus (Drury, 1773) (5/2)

Hesperiidae

$187 \quad$ Nisoniades sp. 1 (1/1) Hesperiidae

$188 \quad$ Nyctelius nyctelius nyctelius (Latreille, 1824) (1/1) Hesperiidae

189 Panoquina lucas (Fabricius, 1793) (4/3) Hesperiidae 


\begin{tabular}{|c|c|}
\hline $\begin{array}{l}\text { Species } \\
\text { number }\end{array}$ & Flower visitors \\
\hline 190 & $\begin{array}{l}\text { Polites vibex catilina (Plötz, 1886) (1/1) } \\
\text { Hesperiidae }\end{array}$ \\
\hline 191 & $\begin{array}{l}\text { Urbanus proteus proteus (Linnaeus, 1758) } \\
\text { Hesperiidae (1/1) }\end{array}$ \\
\hline 192 & Hesperiidae sp. $1(1 / 1)$ \\
\hline 193 & Hesperiidae sp. 2 (1/1) \\
\hline 194 & Hesperiidae sp. $3(2 / 2)$ \\
\hline 195 & Hesperiidae sp. $4(2 / 1)$ \\
\hline 196 & Hesperiidae sp. $5(1 / 1)$ \\
\hline 197 & Emesis sp. 1 (1/1) Lycaenidae \\
\hline 198 & $\begin{array}{l}\text { Albergina vanessoides (Prittwitz, 1865) (1/1) } \\
\text { Lycaenidae }\end{array}$ \\
\hline 199 & Strymon sp. 1 (1/1) Lycaenidae \\
\hline 200 & $\begin{array}{l}\text { Agraulis vanillae maculosa (Stichel, 1907) (2/2) } \\
\text { Nymphalidae }\end{array}$ \\
\hline 201 & $\begin{array}{l}\text { Anarthia amathea roeselia (Eschscholtz, 1821) } \\
\text { (1/1) Nymphalidae }\end{array}$ \\
\hline 202 & $\begin{array}{l}\text { Dryas iulia alcionea (Cramer, 1779) (2/2) } \\
\text { Nymphalidae }\end{array}$ \\
\hline 203 & $\begin{array}{l}\text { Junonia evarete (Cramer, 1779) (9/6) } \\
\text { Nymphalidae }\end{array}$ \\
\hline 204 & $\begin{array}{l}\text { Vanessa braziliensis (Moore, 1883) (5/5) } \\
\text { Nymphalidae }\end{array}$ \\
\hline 205 & $\begin{array}{l}\text { Vanessa myrinna (Doubleday, 1849) (1/1) } \\
\text { Nymphalidae }\end{array}$ \\
\hline 206 & $\begin{array}{l}\text { Yphthimoides celmis (Godart, [1824]) (4/4) } \\
\text { Nymphalidae }\end{array}$ \\
\hline 207 & Nymphalidae sp. $1(1 / 1)$ \\
\hline 208 & $\begin{array}{l}\text { Battus polydamas polydamas (Linnaeus, 1758) } \\
\text { (2/1) Papilionidae }\end{array}$ \\
\hline 209 & $\begin{array}{l}\text { Parides bunichus perrhebus (Boisduval, 1836) } \\
\text { (1/1) Papilionidae }\end{array}$ \\
\hline 210 & $\begin{array}{l}\text { Colias lesbia lesbia (Fabricius, 1775) (2/1) } \\
\text { Pieridae }\end{array}$ \\
\hline 211 & $\begin{array}{l}\text { Rhabdodryas trite banksi (Breyer, 1939) (1/1) } \\
\text { Pieridae }\end{array}$ \\
\hline 212 & Lepidoptera sp. 1 (1/1) \\
\hline 213 & Lepidoptera sp. 2 (1/1) \\
\hline 214 & Lepidoptera sp. 3 (1/1) \\
\hline 215 & Lepidoptera sp. $4(3 / 2)$ \\
\hline 216 & Lepidoptera sp. $5(1 / 1)$ \\
\hline 217 & Lepidoptera sp. $6(1 / 1)$ \\
\hline 218 & Lepidoptera sp. $7(4 / 1)$ \\
\hline 219 & Lepidoptera sp. $8(2 / 2)$ \\
\hline
\end{tabular}

\section{Discussion}

The climate conditions during the dry season are considerably adverse to phenological events, especially by the water restrictions in this period. The climate in
Southern Brazil is seasonal, and in addition to water shortage, the dry season is a period of low temperatures in comparison to other regions of the country. Thus, a great reduction in the number of flowering species in the dry season, as observed in the present study, was expected. The seasonal flowering pattern observed here is in accordance with the flowering data observed in other grassland communities in southeastern Brazil, where the dry and rainy seasons are also well defined (Freitas \& Sazima 2006, Tannus et al. 2006).

According to Peres (2000), seasonality is an important event for the availability of resources, and the seasonality in flowering period would be an important factor to demonstrate the existence of key-species in determined period of the year. According to this criterium, Baccharis rufescens, B. patens, B. cultrata and Croton gnaphalii, large shrubs with many flowers, could be considered key-species in the studied plant community. In the winter, where a low number of flowering species was recorded, these plant species were the exclusive or main resource consumed by insects that were active flyers during cold months, as social bees, social wasps, and some flies.

The prevalence of nectar as the main resource in the study was also recorded in others ecosystems, such as campo rupestre (Faria 1994), cerrado (Barbosa 1997), caatinga (Machado \& Lopes 2004) and high-altitude grasslands (Freitas \& Sazima 2006). In fact, nectar is the main floral resource offered by the plants to their pollen vectors (Proctor et al. 1996) and is the most used floral resource by a great variety of floral visitors (Simpson \& Neff 1981, Endress 1994). The percentage of species offering pollen as the main floral resource was also similar to that recorded in campo rupestre, cerrado, caatinga and hight-altitude grasslands (Faria 1994, Barbosa 1997, Machado \& Lopes 2004, Freitas \& Sazima 2006). However, pollen-flowers in study area were rare in comparison to these other communities, where the families Melastomataceae, Fabaceae, Solanaceae are represented by several species with poricidal anthers. The percentage of flowers offering both pollen and nectar as floral resource (pollen in this class being intentionally collected) was similar to others grassy communities (Barbosa 1997, Freitas \& Sazima 2006) and much higher than that observed in the caatinga (Machado \& Lopes 2004). In this study eight species of oil-flowers were recorded, but oil-bees were recorded only in three plant species from the family Iridaceae and Scrophulariaceae. The percentage of this group of plants was similar to that recorded in high-altitude grasslands (Freitas \& Sazima 2006), in cerrado (Silberbauer-Gottsberger \& Gottsberger 1988), and in the campo rupestre (Faria 1994) but lower 
than the recorded in caatinga where several species of Malpighiaceae and Scrophulariaceae are found (Machado \& Lopes 2004).

In the studied environment, as also recorded in others grassland communities (Barbosa 1997, Freitas \& Sazima 2006), Asteraceae was the family with the highest number of plant species and the most visited by a wide range of floral visitors. In surveys on bee-plant interaction, Asteraceae has been considered by several authors one of the most important sources of floral resources, with the highest number of visited plant species, and the highest richness and abundance of bees recorded (Martins 1995, Schlindwein 1995, Bortoli \& Laroca 1990, Carvalho \& Bego 1997, Alves-dos-Santos 1999, Barbola et al. 2000, Faria-Mucci et al. 2003, Antonini \& Martins 2003). Asteraceae was also found to be the richest, and the most visited family by flies (Arruda \& Sazima 1996, Souza-Silva et al. 2001), and wasps (Hermes \& Köhler 2006). The preference for Asteraceae flowers was probably due to characteristics that make these plants more attractive to floral visitors in comparison to plants in other families: their inflorescences with a large number of flowers were more attractive to floral visitors than scattered single flowers, also serving as landing area for these animals; the floral traits (i.e. floral tube size with few millimeters and the secondary pollen presentation) allow the free access to the resources to a broad range of floral visitors (Endress 1994, Proctor et al. 1996). Moreover, Asteraceae is the largest among angiosperms, and is one of the dominant families in the herbaceous and bushy strata in open habitats (Boldrini 1997, Matzenbacher 2003), similarly to the present study, where this family had the highest number of species.

In this study, the plant taxa with a large number of flowers and with nectar and pollen easily accessed were the most visited by species and individuals of flower visitors. Similarly to the flowers of Asteraceae, the flowers in the families Apiaceae, Arecaceae, and Euphorbiaceae, were also small and had readily accessible resources. In addition, during the flowering period, species of Baccharis, Butia, Croton, and Eryngium produced many flowers grouped in large inflorescences. Such inflorescences could greatly enhance the floral display, attracting different visitors and potential pollinators (Proctor et al. 1996). Thus, the flowers presenting the features mentioned above were usually visited by a wide spectrum of insects, including species with proboscis shorter than those found in bees, such as wasps and flies. So, this kind of flowers can be pollinated by different groups of floral visitor (Endress 1994, Proctor et al. 1996) and are called polyphilic species (Faegri \& van der Pijl 1979). Conversely, plant species with few flowers and floral resources less accessible were less visited, and/or had their floral resources used by more specific floral visitors. Such flowers were found mainly in some species of the families Iridaceae, Oxalidaceae, Fabaceae, Gesneriaceae and Polygalceae.

The number of insect species and individuals recorded followed climate variations, decreasing in the dry season when the conditions were less favorable (i.e. low temperatures), and increasing in the warmest period of the year in the wet season. However, several other factors could influence insect seasonality. The seasonal variation in flower visits is almost certainly related to resource availability (Souza-Silva et al. 2001). In this study, for example, the variation in the number of insects followed the seasonal pattern of flowering in the community, and consequently a decrease or increase in the availability of floral resources may have influenced floral visitors' seasonality. In addition, insect seasonality in a community could be also influenced by reproductive phenology of different species of floral visitors (Wolda 1988).

Since bees depend exclusively on floral resources for their survival, they are the most frequent visitors found on flowers, and were reported as the most diverse and abundant group of floral visitors, as well as the main pollinators in several plant communities (Ramirez 1989, Barbosa 1997, Momose et al. 1998, Oliveira \& Gibbs 2000, Machado \& Lopes 2004, Ramirez 2004, Freitas \& Sazima 2006). Flies and wasps, after bees, were the predominant floral visitors recorded in this study, as well as in cerrado and high-altitude grasslands (Barbosa 1997, Freitas \& Sazima 2006), while beetles and lepidopterans were less represented in all three communities.

Although the goals of this study are not to compare different sampling methods for floral-visitors, the method applied here showed visit frequencies on flowers very similar to the recorded in other plant communities (Faria 1994, Schlindwein 1995, Arruda \& Sazima 1996, Carvalho \& Bego 1997, Alves-dos-Santos 1999, Barbola et al. 2000, Souza-Silva et al. 2001, Faria-Muci et al. 2003, Hermes \& Köhler 2006), where the plant species with floral resources easily accessed, and many flowers, were also the most visited. Thus, resource accessibility and quantity may determine how many floral visitors will be attracted to the flowers. Hence, in an addition to the method, the abundance of flowers could be used as an indicator of resource availability in each plant species in the plant community.

The great richness and the abundance of visits recorded in the Asteraceae flowers, indicated that this family was the main resource used for anthophilous insects, mainly generalist foragers, in this plant community. 
However, the importance of plant species as source of floral resources cannot be measured only by abundance and richness of floral visitors recorded in the flowers. Plant species less frequently visited, for example, may reserve their resources for more specialized floral visitors, maximizing pollination. Thus, these plant species are very important for their visitors, since the exclusion of non effective visitors will decrease the resource competition effect for their legitimate visitors (= pollinators). Moreover, in the community studied, floral oils were recorded in few plants that received few visits. However, these plant species are a very important food source for the maintenance of the diversity of specialized floral visitors such as oil-colleting bees (i.e. Centridini, Tapinotaspidini and Tetrapediini) (Machado \& Lopes 2004, Schlindwein 2000).

The great majority of plant species (ca. 83\%) in the studied community had a floral structure that allowed a free access to the floral resources, such as small size, brush, short-tube (mainly in Asteraceae species), inconspicuous and disc types (Pinheiro 2005). Thus, these plant species were visited by two or more insect groups. Among the 97 plant species visited by bees, for example, $56.6 \%(n=60)$ received, in addition to bees, other groups of floral visitors, mainly wasps and flies. In addition, only $2.9 \%$ and $5.8 \%$ of the plant species visited by wasps and flies, respectively, were not visited by bees, but were visited by other insect groups. Small, open flowers have a larger range of interactions with different species of insects, and are potentially more generalists, than flowers with floral traits that protect them from depletion by forager robbers (Corbet 2006). Thus, in this plant community, the predominance of polyphilic species may result in a considerable degree of generalization between plant-pollinators interactions.

The results of this work highlights the importance of some plant species in terms of supporting a large number of insect visitors and have, consequently, great importance for contribution to the maintenance of insect population and diversity in the community studied. This kind of information can be used as an alternative on habitat management, where is essential to include plants with floral rewards to attract and support pollinator communities.

Aknowledgements - We would like to thank N.I. Matzenbacher, C. Mondin, and A. Schneider for the identification of Asteraceae, M. Sobral for the identification of Myrtaceae, and Lilian Eggers for the identification of Iridaceae. We also thank Danúncia Urban for identification of some bee species, C. Iserhard, L. Kaminski, A.L. Paz for identification of Lepidoptera, M. Hermes for identification of Vespidae, M. Morales for the identification of Syrphidae, R. Moraes for identification of families of Coleoptera, and Laura Utz for technical assistance with the English. FAPERGS, Capes, CNPq and FBPN-MacArthur Foundation are thanked for essential financial support. Special thanks to two anonymous referees for their valuable suggestions.

\section{References}

AGUIAR, C.M.L. 2003. Utilização de recursos florais por abelhas (Hymenoptera, Apoidea) em uma área de Caatinga (Itatim, Bahia, Brasil). Revista Brasileira de Zoologia 20:457-467.

ALVES-DOS-SANTOS, I. 1999. Abelhas e plantas melíferas da Mata Atlântica, restinga e dunas do litoral norte do Estado do Rio Grande do Sul, Brasil. Revista Brasileira de Entomologia 43:191-223.

ANGIOSPERM PHYLOGENY GROUP II. 2003. An update of the Angiosperm Phylogeny Group classification for the ordens and families of flowering plants. Botanical Journal of Linnean Society 141:399-436.

ANTONINI, Y. \& MARTINS, R.P. 2003. The floweringvisiting bees at the Ecological Station of the Universidade Federal de Minas Gerais, Belo Horizonte, MG, Brazil. Neotropical Entomology 32:565-575.

ARRUDA, V.L.V. \& SAZIMA, M. 1996. Flores visitadas por sirfídeos (Diptera: Syrphidae) em uma mata mesófila de Campinas, SP. Revista Brasileira de Botânica 19: 109-117.

BARBOLA, I., LAROCA, S. \& ALMEIDA, M.C. 2000. Utilização de recursos florais por abelhas silvestres (Hymenoptera, Apoidea) da Floresta Estadual Passa Dois (Lapa, Paraná, Brasil). Revista Brasileira de Entomologia 44:9-19.

BARBOSA, A.A.A. 1997. Biologia reprodutiva de uma comunidade de campo sujo, Uberlândia, MG. Tese de doutorado, Universidade Estadual de Campinas, Campinas.

BAWA, K.S. 1990. Plant-pollinator interactions in tropical rain forests. Annual Review of Ecology and Systematics 21:399-422.

BOLDRINI, I.I. 1997. Campos do Rio Grande do Sul: caracterização fisionômica e problemática ocupacional. Boletim do Instituto de Biociências 56:1-39.

BORTOLI, C. \& LAROCA, S. 1990. Estudo biocenótico em Apoidea (Hymenoptera) de uma área restrita em São José dos Pinhais (PR, sul do Brasil), com notas comparativas. Dusenia 15:1-112.

CAMARGO, J.M.F. \& MAZUCATO, M. 1984. Inventário da apifauna e flora apícola de Ribeirão Preto, SP, Brasil. Dusenia 14:55-87.

CARPENTER, J.M. \& MARQUES, O.M. 2001. Contribuição ao estudo dos vespídeos do Brasil (Insecta, Hymenoptera, Vespoidea, Vespidae). Série Publicações Digitais, Universidade Federal da Bahia, v.2, [CD-ROM]. 
CARVALHO, A.M.C. \& BEGO, L. 1997. Exploitation of available resources by bee fauna (Apoidea-Hymenoptera) in the Reserva Ecológica do Panga, Uberlândia, State of Minas Gerais, Brazil. Revista Brasileira de Entomologia 41:101-107.

CORBET, S.A. 2006. A typology of pollination systems: implications for crop management and the conservation of wild plants. In Plant-pollinator interactions (N.M. Waser \& J. Ollerton, eds.). The University of Chicago Press, Chicago, p.314-340.

CORRÊA, C.A., IRGANG, B.E. \& MOREIRA, G.R.P. 2001. Estrutura floral das angiospermas usadas por Heliconius erato phyllis (Lepidoptera, Nymphalidae) no Rio Grande do Sul. Iheringia, série Zoológica 90: 71-84.

DARRAULT, R.O. \& SCHLINDWEIN, C. 2002. Esfingídeos (Lepidoptera, Sphingidae) no Tabuleiro Paraibano, nordeste do Brasil: abundância, riqueza e relação com plantas esfingófilas. Revista Brasileira de Zoologia 19:429-443.

ENDRESS, P.K. 1994. Diversity and evolutionary biology of tropical flowers. Cambridge University Press, Cambridge.

ENNES, R.W. 1956. A revision of the genera Nemognatha, Zonitis and Pseudozonitis (Coleoptea, Meloideae) in America north of Mexico, with a proposed new genus. University Kansas Science Bulletin 37:985-905.

FAEGRI, K. \& VAN DER PIJL, L. 1979. The principles of pollination ecology. Pergamon Press, Oxford.

FARIA, G.M. 1994. A flora e a fauna apícola de um ecossistema de campo rupestre, Serra do Cipó, MG, Brasil: composição, fenologia e suas interações. Tese de doutorado, Universidade Estadual de Rio Claro, Rio Claro.

FARIA-MUCCI, G.M., MELO, M.A. \& CAMPOS, L.A.O. 2003. A fauna de abelhas (Hymenoptera, Apoidea) e plantas utilizadas como fonte de recursos florais, em um ecossistema de campos rupestres em Lavras Novas, Minas Gerais, Brasil. In Apoidea Neotropica: Homenagem aos 90 anos de Jesus Santiago Moure (G.A.R. Melo \& I. Alves-dos-Santos, eds.). Editora Unesc, Criciúma, p.241-256.

FREITAS, L. \& SAZIMA, M. 2006. Pollination biology in a tropical high-altitude grassland in Brazil: interactions at the community level. Annals of Missouri Botanical Garden 93:465-516.

HEITHAUS, E.R. 1974. The role of plant-pollinator interactions in determining community struture. Annals of the Missouri Botanical Garden 61:675-691.

HERMES, M.G. \& KÖHLER, A. 2006. The flower-visiting social wasps (Hymenoptera, Vespidae, Polistinae) in two areas of Rio Grande do Sul State, Southern Brazil. Revista Brasileira de Entomologia 50:268-274.

KÖPPEN, W. 1948. Climatología: con un estudio de los climas de la tierra. Fundo de Cultura Econômica, México.
LAMAS, G. 2004. Atlas of Neotropical Lepidoptera: checklist part 4A (Hesperioidea-Papilionoidea). Scientific Publishers, Gainesville.

LORENZON, M.C.A., MATRANGOLO, C.A.R. \& SCHOEREDER, J.H. 2003. Flora visitada pelas abelhas eussociais (Hymenoptera, Apidae) na Serra da Capivara, em Caatinga do Sul do Piauí. Neotropical Entomology 32:27-36.

MACHADO, I.C. \& LOPES, A.V. 2004. Floral traits and pollination systems in the caatinga, a Brazilian tropical dry forest. Annals of Botany 94:365-376.

MARINONI, L., MORALES, M.N., \& SPALER, I. 2007. Chave de identificação ilustrada para os gêneros de Syrphinae (Diptera, Syrphidae) de ocorrência no sul do Brasil. Biota Neotropica 7:143-158.

MARTINS, C.F. 1995. Flora apícola e nichos tróficos de abelhas (Hym., Apoidea) na Chapada Diamantina (Lençóis-BA, Brasil). Revista Nordestina de Biologia 10:119-140.

MATZENBACHER, N.I. 2003. Diversidade florística dos campos sul-brasileiros: Asteraceae. In Desafios da botânica brasileira no novo milênio: inventário, sistematização e conservação da diversidade vegetal (M.A.G, Jardim, M.N.C, Bastos \& J.U.M, Santos, eds.). MPEG, Belém, p.124-127.

MOMOSE, K., YUMOTO, T., NAGAMITSU, T., KATO, M., NAGAMASU, H., SAKAI, S., HARRISON, R.D., ITIOKA, T., HAMID, A.A. \& INOUE, T. 1998. Pollination biology in a lowland dipterocarp forest in Sarawak, Malaysia. I. Characteristics of the plantpollinator community in a lowland dipterocarp forest. American Journal of Botany 85:1477-1501.

NEWSTRON, L.E., FRANKIE, G.W. \& BAKER, H.G. 1994. A new classification for plant phenology based on flowering patterns in Low Tropical Rain Forest Trees at La Selva, Costa Rica. Biotropica 26:141-159.

OLIVEIRA, P.E. \& GIBBS, P.G. 2000. Reproductive biology of woody plants in a cerrado community of Central Brazil. Flora 195:311-329.

OLIVEIRA, P.E., GIBBS, P.E. \& BARBOSA, A.A. 2004. Moth pollination of woody species in the Cerrados of central Brazil: a case of so much owed to so few? Plant Systematics and Evolution 245:41-52.

PERES, C.A. 2000. Identifying keystone plant resources in tropical forests: the case of gums from Parkia pods. Journal of Tropical Ecology 16:287-317.

PINHEIRO, M. 2005. Fontes de recursos florais e biologia da polinização em uma comunidade campestre no Sul do Brasil. Tese de Doutorado, Universidade Federal do Rio Grande do Sul, Porto Alegre.

PRANCE, G.T. 1990. Management and conservation of tropical ecosystems requires knowledge of plant-animal interactions: afterword. Memoirs of the New York Botanical Garden 55:186-187.

PROCTOR, M., YEO, P. \& LACK, A. 1996. The natural history of pollination. Harper Collins Publishers, London. 
RAMIREZ, N. 1989. Biologia de polinização en una comunidad arbustiva tropical de la Alta Guayana Venezolana. Biotropica 21:319-330.

RAMIREZ, N. 2004. Pollination specialization and time of pollination on a tropical Venezuelan plain: variations in the time and space. Botanical Journal of Linnean Society 145:1-16.

SCHLINDWEIN, C. 1995. Wildbienen und ihre Trachtpflanzen in einer südbrasilianischen Buschlandschaft: Fallstudie Guaritas, Bestäubung bei Kakteen und Loasaceen. Verlag Ulrich E. Grauer, Stuttgard.

SCHLINDWEIN, C. 1998. Frequent oligolecty characterizing a diverse bee-plant community in a xerophytic bushland of subtropical Brazil. Studies on Neotropical Fauna and Environment 33:46-59.

SCHLINDWEIN, C. 2000. A importância de abelhas especializadas na polinização de plantas nativas e conservação do meio ambiente. In Anais do IV Encontro sobre Abelhas. Ribeirão Preto, p.131-141.

SECRETARIA ESTADUAL DO MEIO AMBIENTE. 1997. Plano de manejo do Parque Estadual de Itapuã. Secretaria Estadual do Meio Ambiente, Porto Alegre.

SILBERBAUER-GOTTSBERGER, I. \& GOTTSBERGER, G. 1988. A polinização de plantas do Cerrado. Revista Brasileira de Biologia 48:651-663.

SILVEIRA, F.A., MELO, G.A.R. \& ALMEIDA, E.A.B. 2002. Abelhas brasileiras: sistemática e identificação. Ministério do Meio Ambiente/Fundação Araucária, Belo Horizonte.
SIMPSON, B.B. \& NEFF, J.L. 1981. Floral rewards: alternatives to pollen and nectar. Annals of the Missouri Botanical Garden 68:301-322.

SOUZA-SILVA, M., FONTENELLE, J.C.R. \& MARTINS, R.P. 2001. Seasonal abundance and species composition of flower-visiting flies. Neotropical Entomology 30: 351-359.

TANNUS, J.L.S., ASSIS, A.M. \& MORELLATO, L.P.P.C. 2006. Fenologia reprodutiva em campo sujo e campo úmido numa área de cerrado no sudeste do Brasil. Biota Neotropica 6:1-23.

TRIPLEHORN, C.A. \& JOHNSON, N.F. 2005. Borror \& DeLong's introduction to the study of insects. $7^{\text {th }}$ ed., Brooks Cole, Belmont.

UNIVERSIDADE FEDERAL DO RIO GRANDE DO SUL. 1982. Itapuã, análise preliminar do espaço geográfico. Editora da Universidade Federal do Rio Grande do Sul, Porto Alegre.

VIANA, B.F., SILVA, F.O. \& KLEINERT, A.M.P. 2006. A flora apícola de uma área de restinga de dunas litorâneas, Abaeté, Salvador, Bahia. Revista Brasileira de Botânica 29:13-25.

WILMS, W., WENDEL, L., ZILLIKENS, A., BLOCHTEIN, B. \& ENGELS, W. 1997. Bees and other insects recorded on flowering trees in a subtropical Araucaria forest in southern Brazil. Studies on Neotropical Fauna and Environment 32:220-226.

WOLDA, H. 1988. Insect seasonality: why? Annual Review of Ecology and Systematics 19:1-18. 